\title{
Schaffung von grenzübergreifenden Versorgungsstrukturen in der Unfallversicherung
}

\author{
Eva-Marie Höffer
}

$\begin{array}{ll}\text { A. Einleitung } & 269\end{array}$

B. Arbeitnehmerfreizügigkeit und Dienstleistungsfreiheit 270

I. Entwicklung der Beschäftigungszahlen $\quad 271$

II. Demographie und Fachkräftemangel 272

C. Sozialversicherungsrechtliche Beurteilung von Beschäftigungen polnischer Staatsangehöriger in Deutschland 273

I. Beschäftigungslandprinzip 273

II. Entsendung 274

III. Mehrfachbeschäftigung 275

IV. Verfahren der Sachleistungsaushilfe $\quad 276$

V. Praktische Erfahrungen 276

D. Charakteristika der gesetzlichen Unfallversicherung in Deutschland 278

E. Grenzübergreifende Versorgungsstrukturen 279

F. Regelungen im Europarecht 280

I. Regelungen des koordinierenden Verordnungsrechts 281

II. Patientenrichtlinie 281

G. Anforderungen an die Umsetzung 282

H. Fazit 282

\section{A. Einleitung}

Seit dem 1. Mai 2011 gilt für polnische Staatsangehörige, die in Deutschland eine Beschäftigung aufnehmen möchten, die uneingeschränkte Arbeitnehmerfreizügigkeit.

Es stellt sich die Frage, welche sozialversicherungsrechtlichen Auswirkungen, insbesondere in Bezug auf die gesetzliche Unfallversicherung in Deutschland, hiermit verbunden sind. Dabei ist unter anderem von Interesse, ob die uneingeschränkte Arbeitnehmerfreizügigkeit zu einem Anstieg an Beschäftigungsverhältnissen in Deutschland führen wird. Konjunkturelle Entwicklungen und Trends auf dem Arbeitsmarkt können sich auf das System der gesetzlichen Unfallversicherung auswirken, das durch Unter- 
nehmensbeiträge finanziert ist, bei deren Berechnung die Arbeitsentgelte maßgebend sind ( $\S 150,153$ SGB VII). Polen ist unter den acht mittel- und osteuropäischen Mitgliedstaaten, für die die Arbeitnehmerfreizügigkeit seit dem 1. Mai 2011 unbeschränkt gilt ${ }^{1}$, aus Sicht der gesetzlichen Unfallversicherung in mehrfacher Hinsicht von besonderer Bedeutung. Polen und Deutschland haben eine gemeinsame Grenze. Darüber hinaus haben in Bezug auf die acht mittel- und osteuropäischen Staaten polnische Staatsbürger bereits vor der vollständigen Öffnung des deutschen Arbeitsmarktes die meisten Arbeitsgenehmigungen für die Aufnahme einer Beschäftigung in Deutschland erhalten. ${ }^{2}$ Neben den deutsch-polnischen Kontakten der auf dem Gebiet der Unfallversicherung zuständigen Verbindungsstellen besteht zudem seit einigen Jahren ein regelmäßiger deutsch-polnischer Austausch zur medizinischen Versorgung Unfallverletzter, der zu dem Gedanken eines grenzübergreifenden Versorgungskonzeptes zur medizinischen Versorgung in der Rehabilitationsphase geführt hat.

\section{B. Arbeitnehmerfreizügigkeit und Dienstleistungsfreiheit}

Arbeitnehmerfreizügigkeit und Dienstleistungsfreiheit sind für die gesetzliche Unfallversicherung in Deutschland vor allem in Bezug auf die Beschäftigung polnischer Arbeitskräfte in Deutschland relevant. Die Tätigkeit selbständig erwerbstätiger Polen in Deutschland erlangt in Bezug auf den Gesundheits- und Pflegebereich ebenfalls Bedeutung.

Angehörige der Europäischen Mitgliedstaaten können innerhalb der Europäischen Union (EU) frei wählen, in welchem Mitgliedstaat sie leben und eine Beschäftigung aufnehmen möchten. Dies ermöglicht die im EU-Vertrag verankerte Arbeitnehmerfreizügigkeit (Art. 45 des Vertrags über die Arbeitsweise der EU - AEUV). Die Mobilität von Arbeitskräften innerhalb Europas ist damit ein wesentlicher Bestandteil des europäischen Binnenmarktes. Das Recht auf Freizügigkeit war bis zum 1. Mai 2011 für Staatsangehörige aus acht mittel- und osteuropäischen Staaten, die am 1. Mai 2004 der EU beigetreten waren, in Bezug auf die Aufnahme von Beschäftigungen in Deutschland beschränkt. Die Aufnahme einer Beschäftigung in Deutschland war für die Staatsbürger dieser Länder und damit auch für polnische Staatsbürger von einer Arbeitsgenehmigung der EU abhängig. Für Akademiker wurden diese Beschränkungen bereits im Jahre 2009 aufgehoben. ${ }^{3}$

1 Seit dem 1.Mai 2011 ist der deutsche Arbeitsmarkt vollständig für Staatsbürger aus Polen, Ungarn, der Tschechischen Republik, der Slowakei, Estland, Lettland und Litauen geöffnet.

2 Vgl. Statistik der Bundesagentur für Arbeit Arbeitsmarkt in Zahlen, Arbeitsgenehmigungen-EU/Zustimmungen, Berichtsjahre 2009 und 2010.

3 Bundesagentur für Arbeit (Hrsg.), Welle an Billigjobbern ist ausgeblieben - Zwischenbilanz zur Neuregelung der Arbeitnehmerfreizügigkeit, Presse Info 010/2012 vom 13.01.2012. 
Neben der Arbeitnehmerfreizügigkeit hat Deutschland ebenfalls übergangsweise die Ausübung von Dienstleistungen und damit die Dienstleistungsfreiheit (Art. 56 AEUV) für bestimmte, sensible Sektoren (Bau, Gebäudereinigung und Innendekorateure) beschränkt. ${ }^{4}$ Im Zuge dieser Schutzklauseln war es polnischen Unternehmen dieser Branchen nicht möglich, Dienstleistungen in Deutschland anzubieten und insofern auch nicht, Mitarbeiter zu entsenden. Diese Begrenzung besteht seit dem 1. Mai 2011 ebenfalls nicht mehr. Damit können polnische Selbständige auch aus dem Baubereich nunmehr in Deutschland tätig sein.

Polnische Arbeitnehmer haben bereits vor dem 1. Mai 2011 berufliche Tätigkeiten in Deutschland ausgeübt. Die Tätigkeiten konzentrieren sich auf bestimmte Branchen, insbesondere den Pflege- und den Gesundheitssektor, den Baubereich, das Hotel- und Gaststättengewerbe, die Landwirtschaft und Tätigkeiten im Haushalt. Für die Unfallversicherungsträger ist dabei eine maßgebende Frage, ob ein Beschäftigungsverhältnis vorliegt. Hiervon hängt $a b$, ob eine Versicherungspflicht in der gesetzlichen Unfallversicherung besteht.

Sollte die uneingeschränkte Arbeitnehmerfreizügigkeit zu einem Anstieg an Beschäftigungsverhältnissen polnischer Staatsbürger in Deutschland führen, so hätte diese Entwicklung Einfluss auf die Beiträge zur gesetzlichen Unfallversicherung sowie auf die Leistungen, die die Unfallversicherungsträger nach Eintritt eines Arbeitsunfalls erbringen. Eine derartige Entwicklung kann ebenfalls die Präventionsaktivitäten der Unfallversicherungsträger beeinflussen, etwa indem diese spezielle Angebote entwickeln.

\section{Entwicklung der Beschäftigungszahlen}

Bis zum 1. Mai 2011 war der Zugang zum deutschen Arbeitsmarkt für polnische Beschäftigte von einer Arbeitsgenehmigung abhängig. Den Statistiken der Bundesagentur für Arbeit „Arbeitsmarkt in Zahlen - Arbeitsgenehmigungen und Zustimmungen“ aus den Jahren 2009 und 2010 zufolge, wurden im Jahr 2009 insgesamt 22.585 Arbeitsgenehmigungen für erstmalige Beschäftigungsverhältnisse erteilt, 10.102 Genehmigungen für eine erneute Beschäftigung und 7.890 Genehmigungen in Bezug auf die Fortführung einer Beschäftigung 5 . Diese Zahlen beinhalten nicht die Arbeitserlaubnisverfahren der zentralen Auslands- und Fachvermittlung (ZAV) in Bezug auf Saisonarbeitnehmer, Schausteller und Haushaltshilfen. Im Jahr 2010 wurden 18.265 erstmalige Genehmigungen, 3.471 Arbeitsgenehmigungen für eine erneute Beschäftigung und 3.377 Genehmigungen zur Fortsetzung einer Beschäftigung erteilt. ${ }^{6}$

4 Schannor, Gegen den Missbrauch der Dienstleistungsfreiheit, Die BG 2007, S. 441, 442.

5 Statistik der Bundesagentur für Arbeit Arbeitsmarkt in Zahlen, Arbeitsgenehmigungen-EU/Zustimmungen, Berichtsjahr 2009.

6 Statistik der Bundesagentur für Arbeit Arbeitsmarkt in Zahlen, Arbeitsgenehmigungen-EU/Zustimmungen, Berichtsjahr 2010. 
Absolut gesehen sank die Zahl der erteilten Arbeitsgenehmigungen im Jahr 2010 gegenüber 2009. Allerdings bestand für Akademiker bereits ab dem Jahr 2009 Freizügigkeit.

Im Gesamtvergleich mit den anderen der EU-8-Staaten, für die nunmehr die uneingeschränkte Arbeitnehmerfreizügigkeit gilt, stand Polen sowohl im Jahr 2009 als auch im Jahr 2010 an der Spitze erteilter Arbeitsgenehmigungen sowie Arbeitserlaubnisverfahren, gefolgt von Rumänien.

Die Entwicklung der von der Bundesagentur für Arbeit veröffentlichten Zahlen in Bezug auf sozialversicherungspflichtige Beschäftigungsverhältnisse gibt Aufschluss über deren Entwicklung in den Jahren 2007 bis 2011. Hier zeigt sich in einem Vergleich ein deutlicher Anstieg an sozialversicherungspflichtigen Beschäftigungsverhältnissen im Jahr 2011.7 So stieg die Zahl versicherungspflichtiger Beschäftigungsverhältnisse polnischer Staatsbürger von 83.699 im Jahr 2007 über 88.242 in 2008 über 95.297 in 2009 und $101.834 \mathrm{im} \mathrm{Jahr} 2010$ auf $129.651 \mathrm{im}$ Jahr 2011. Der Anstieg von 2010 auf 2011 war mit 27.817 Beschäftigungsverhältnissen im Vergleich deutlich höher als in den Vorjahren. Inwieweit diese Entwicklung durch den Wegfall der Übergangsfristen beeinflusst war, lässt sich mangels statistischer Daten hierzu nicht belegen.

Einer Presseinformation der Bundesagentur für Arbeit von Januar 2012 in Bezug auf alle acht mittel- und osteuropäischen Mitgliedstaaten zufolge waren Ende Mai 2011 insgesamt 186.000 Personen sozialversicherungspflichtig beschäftigt und 73.000 geringfügig beschäftigt. Aufgrund der uneingeschränkten Arbeitnehmerfreizügigkeit ist danach ein Anstieg der sozialversicherungspflichtigen Beschäftigungen um 15.000 und der geringfügigen Beschäftigungen um $9.000 \mathrm{zu}$ verzeichnen. ${ }^{8}$ Insgesamt kann also in Bezug auf die acht Mitgliedstaaten, darunter auch Polen, festgestellt werden, dass die uneingeschränkte Arbeitnehmerfreizügigkeit sich durch eine Zunahme an Beschäftigungsverhältnissen auf dem deutschen Arbeitsmarkt auswirkt.

\section{Demographie und Fachkräftemangel}

Für Deutschland ist die Gewinnung von Fachkräften aus dem Ausland angesichts der demographischen Entwicklung sowie eines bereits heute bestehenden Fachkräftemangels von besonderer Bedeutung. Polnische Arbeitskräfte, die in Deutschland eine Beschäftigung aufnehmen, sind vor diesem Hintergrund für den deutschen Arbeitsmarkt von Interesse.

7 Statistik der Bundesagentur für Arbeit, Beschäftigungsstatistik, Sozialversicherungspflichtig Beschäftigte nach Staatsangehörigkeiten und Geschlecht, Januar 2012.

8 Bundesagentur für Arbeit Presse Info 010/2012 vom 13.1.2012, „Welle an Billigjobbern ist ausgeblieben - Zwischenbilanz zur Neuregelung der Arbeitnehmerfreizügigkeit“, Presse Info 042/2011. Erste Ergebnisse zu Auswirkungen der uneingeschränkten Arbeitnehmerfreizügigkeit seit dem 1. Mai. 
Deutschland ist jedoch nicht der einzige europäische Staat, in dem der Anteil älterer Menschen im Vergleich zu jüngeren zunimmt. Arbeitskräfte insbesondere aus dem europäischen Ausland werden deswegen den Arbeitskräftemangel nicht ausgleichen. Die gesetzliche Unfallversicherung beschäftigt sich vor diesem Hintergrund mit den Herausforderungen, die Demographie und Fachkräftemangel an die Arbeitswelt stellen. Dabei stehen die Sensibilisierung der Unternehmen, die Förderung einer Mischung aus jungen und älteren Mitarbeitern sowie Präventionsangebote, die ältere Menschen länger in Arbeit gesund halten, im Vordergrund. 9

Demographische Entwicklung und Fachkräftemangel greifen angesichts eines zunehmenden Bedarfs für die Pflege älterer Menschen und eines Fachkräftemangels in Pflegeberufen insbesondere in der Pflege ineinander ${ }^{10}$. In Bezug auf polnische Pflegekräfte, die in Deutschland tätig sind, sind die Unfallversicherungsträger mit einer Reihe von Fragen insbesondere zur versicherungsrechtlichen Einordnung der jeweiligen Tätigkeit befasst.

\section{Sozialversicherungsrechtliche Beurteilung von Beschäftigungen polni- scher Staatsangehöriger in Deutschland}

Die Freizügigkeit im Sinne des Art. 45 AEUV basiert auf dem Grundgedanken, dass jeder Angehörige eines europäischen Mitgliedstaats frei wählen kann, in welchem Mitgliedstaat er lebt und arbeitet. Ein polnischer Staatsangehöriger kann danach in Deutschland beschäftigt sein und auch in Deutschland wohnen oder in Deutschland arbeiten und in Polen wohnhaft bleiben. Es stellt sich aus Sicht der gesetzlichen Unfallversicherung die Frage, welches Sozialversicherungsrecht in derartigen Fällen Anwendung findet.

\section{Beschäftigungslandprinzip}

Die Vorschriften des Sozialgesetzbuches und damit auch die Vorschriften für die gesetzliche Unfallversicherung, die im SGB VII niedergelegt sind, finden auf alle Beschäftigungen und selbständige Tätigkeiten im Geltungsbereich des Gesetzes Anwendung (§ 3 SGB IV i.V.m. § 30 SGB I). Grundsätzlich gilt also, dass eine Person, die in Deutschland beschäftigt ist oder eine selbständige Tätigkeit ausübt, den Vorschriften des Sozialgesetzbuches unterliegt. Nach den Vorschriften des SGB IV bleiben die Bestimmungen des über- und zwischenstaatlichen Rechts unberührt (§ 6 SGB IV). Die

9 Schmid, Alternde Belegschaften und Fachkräftemangel, Der Sicherheitsingenieur 06/2010, S. 40 ff.

10 GVG (Hrsg.), Umgang mit dem Fachkräftemangel in der Pflege, GVG Schriftenreihe Bd. 69, 2011; BGW (Hrsg.), Sieht die Pflege bald alt aus?, BGW Pflegereport 2007, Stand 08/2007. 
Verordnung (EG) Nr. 883/04 zur Koordinierung der Sozialversicherungssysteme hat als überstaatliches Recht Vorrang vor den Vorschriften des deutschen Sozialgesetzbuches. Darin ist auch geregelt, welches Sozialversicherungsrecht in Bezug auf Beschäftigungsverhältnisse anzuwenden ist.

Die Grundregel enthält Art. 11 der Verordnung (EG) Nr. 883/04. ${ }^{11}$ Nach Art. 11 Absatz 1 findet immer nur das Recht eines Mitgliedstaates auf eine Person Anwendung und zwar in Bezug auf alle Zweige der Sozialversicherung. ${ }^{12}$ Gemäß Art. 11 Absatz 3 a) findet das Sozialversicherungsrecht des Mitgliedstaates Anwendung, in dem die Beschäftigung erfolgt. Ist ein polnischer Staatsbürger in Deutschland beschäftigt, findet deutsches Sozialversicherungsrecht Anwendung, auch wenn der Beschäftigte weiterhin in Polen wohnt. Dies gilt auch dann, wenn das Unternehmen seinen Sitz nicht in Deutschland, sondern in Polen oder einem anderen europäischen Mitgliedstaat hat. ${ }^{13}$ Die Anwendung deutschen Sozialversicherungsrechts hat zur Folge, dass die Regelungen der gesetzlichen Kranken-, Pflege-, der Renten-, der Unfall- sowie der Arbeitslosenversicherung gelten.

Gemäß Art. 11 Absatz 3 a) der Verordnung (EG) Nr. 883/04 gilt für Selbständige das Sozialversicherungsrecht des Mitgliedstaates, in dem sie die selbständige Erwerbstätigkeit ausüben. Üben polnische Staatsbürger eine selbständige Erwerbstätigkeit in Deutschland aus, so gelten die Vorschriften des deutschen Sozialversicherungsrechts. Insbesondere in Pflege- und Gesundheitsberufen sowie in Haushalten sind polnische selbständig Erwerbstätige zu finden. Gemäß § 2 Abs. 1 Nr. 9 SGB VII sind Personen, die selbständig oder unentgeltlich im Gesundheitswesen oder in der Wohlfahrtspflege tätig sind, kraft Gesetz gegen Arbeitsunfälle und Berufskrankheiten versichert. Auch selbständig Erwerbstätige in der Altenpflege oder Betreiber ambulanter Pflegedienste sind demnach pflichtversichert. ${ }^{14}$

Von dem beschriebenen Prinzip, wonach die Ausübung der Beschäftigung bzw. selbständigen Erwerbstätigkeit in Deutschland entscheidend für die Anwendbarkeit deutschen Sozialversicherungsrechts ist, gibt es Ausnahmen.

\section{Entsendung}

Eine Entsendung im Sinne des Art. 12 Absatz 1 der Verordnung (EG) Nr. 883/04 liegt vor, wenn eine Person, die in einem Mitgliedstaat auf Rechnung eines Arbeitge-

11 Steinmeyer, in: Fuchs (Hrsg.), Europäisches Sozialrecht, Nomos Kommentar, 5. Aufl. 2010, Art. 11 Rdnr. 1.

12 Ebd.

13 Lauterbach/Platz, in: Breuer (Hrsg.), Unfallversicherung (SGB VII), 4. Aufl. 2011, § 150 Rdnr. 13; die Deutsche Gesetzliche Unfallversicherung (DGUV) hat hierzu Informationen für ausländische Arbeitgeber entwickelt, die im Internet erhältlich sind: http://www.dguv.de, Webcode: d108809.

14 Berufsgenossenschaft für Gesundheit und Wohlfahrtspflege, Merkblatt zur gesetzlichen Unfallversicherung für die in der Nachbarschaftshilfe, Alten-, Haus- und Familienpflege tätigen Personen. 
bers, der gewöhnlich dort tätig ist, eine Beschäftigung ausübt, von diesem Arbeitgeber in einen anderen Mitgliedstaat entsandt wird, um dort eine Arbeit für dessen Rechnung auszuführen. In diesem Fall unterliegt sie weiterhin den Rechtsvorschriften des ersten Mitgliedstaats, sofern die voraussichtliche Dauer dieser Arbeit 24 Monate nicht überschreitet und diese Person nicht eine andere Person ablöst.

Wird ein polnischer Arbeitnehmer von seinem gewöhnlich in Polen tätigen Arbeitgeber nach Deutschland entsandt, um dort für einen Zeitraum, der 24 Monate nicht überschreitet, eine Arbeit für dessen Rechnung auszuführen, so gelten weiterhin die Vorschriften des polnischen Sozialversicherungsrechts. Führt der polnische Arbeitnehmer die Bescheinigung A 1 mit sich, in der die Weitergeltung der polnischen Sozialversicherungsvorschriften bescheinigt ist, so sind die deutschen Unfallversicherungsträger hieran gebunden, es sei denn die Bescheinigung wird von der ausstellenden Behörde zurückgenommen (Art. 5 Verordnung (EG) Nr. 987/09). ${ }^{15}$

Die Regelungen der Entsendung finden auch auf Selbständige Anwendung (Art. 12 Abs. 2 Verordnung (EG) Nr. 883/2004). Voraussetzung ist die Ausübung einer entsprechenden Tätigkeit. Auf eine selbständige Pflegekraft aus Polen, die in Deutschland eine pflegende selbständige Erwerbstätigkeit ausübt, die 24 Monate nicht überschreitet, finden danach weiterhin die polnischen Vorschriften der Sozialversicherung Anwendung.

\section{Mehrfachbeschäftigung}

Nach Art. 13 Abs. 1 a) der Verordnung (EG) Nr. 883/2004 unterliegt eine Person, die gewöhnlich in zwei oder mehr Mitgliedstaaten eine Beschäftigung ausübt, dann den Rechtsvorschriften des Wohnstaates, wenn sie dort einen wesentlichen Teil ihrer Tätigkeit ausübt oder wenn sie bei mehreren Unternehmen oder Arbeitgebern beschäftigt ist, die ihren Sitz in verschiedenen Mitgliedstaaten haben. In letzterem Fall kann es sich auch um mehrere geringfügige Beschäftigungsverhältnisse handeln.

Arbeitet danach ein polnischer Beschäftigter mit Wohnsitz in Polen gewöhnlich in Polen und Deutschland und übt er in Polen einen wesentlichen Teil seiner Beschäftigung aus, findet polnisches Sozialversicherungsrecht Anwendung (Art. 13 Absatz 1 a)). ${ }^{16}$ Wenn der polnische Beschäftigte in Deutschland wohnt und dort auch den wesentlichen Teil seiner Beschäftigung ausübt, findet deutsches Sozialversicherungsrecht Anwendung.

Arbeitet ein polnischer Beschäftigter gewöhnlich in Polen und Deutschland für verschiedene Arbeitgeber, ist der Wohnsitz entscheidend (Art. 13 Absatz 1 a)). Wohnt der polnische Beschäftigte in Deutschland, finden die Vorschriften der gesetzlichen Unfall-

15 EuGH-Urteil v. 10.2.2000, Rs. C-202/97 (Fitzwilliam Technical Services), Slg. 2000, I-883; v. 26.1.2006, Rs. C-2/05 (Herbosch Kiere NV), Slg. 2006, I-1079; s. Art. 5 VO (EG) 987/09.

16 Zur Problematik der Abgrenzung zur Entsendung sowie zu der Frage, wann das Kriterium eines wesentlichen Teils der Beschäftigung erfüllt ist: Steinmeyer (Fußn. 11), Art. 13 Rdnr. 9, 10. 
versicherung in Deutschland auch in Bezug auf das Beschäftigungsverhältnis in Polen Anwendung. Wohnt der Beschäftigte in Polen, findet insgesamt polnisches Sozialversicherungsrecht Anwendung.

In der Praxis sind des Weiteren Fallkonstellationen anzutreffen, in denen eine Beschäftigung mit einer selbständigen Tätigkeit zusammentrifft. Übt ein in Polen Beschäftigter gleichzeitig eine selbständige Tätigkeit in Deutschland aus, findet das Sozialversicherungsrecht des Landes Anwendung, in dem die Beschäftigung ausgeübt wird, in diesem Fall polnisches Sozialversicherungsrecht (Art. 13 Absatz 3). Ist ein in Polen selbständig Erwerbstätiger gleichzeitig in Deutschland abhängig beschäftigt, gilt deutsches Sozialversicherungsrecht.

Übt ein in Polen selbständig Erwerbstätiger gleichzeitig eine selbständige Erwerbstätigkeit in Deutschland aus, gelten die Vorschriften des Wohnstaates, soweit dort ein wesentlicher Teil der Tätigkeit ausgeübt wird (Art. 13 Absatz 2 a)). Ob deutsches oder polnisches Sozialversicherungsrecht zur Anwendung kommt, hängt danach davon $a b$, ob sich der Wohnsitz in Polen oder in Deutschland befindet.

\section{Verfahren der Sachleistungsaushilfe}

Ist bei einer Entsendung aus Polen oder in Bezug auf eine Mehrfachbeschäftigung polnisches Sozialversicherungsrecht anzuwenden und tritt während der Tätigkeit in Deutschland ein Arbeitsunfall ein, so erhält der Verletzte über das Verfahren der Sachleistungsaushilfe Leistungen nach den Grundsätzen und Verfahren der gesetzlichen Unfallversicherung auf Rechnung des zuständigen polnischen Trägers (Art. 36 Abs. 1 und 2 Verordnung (EG) Nr. 883/04). ${ }^{17}$ Mit den Aufgaben der Sachleistungsaushilfe und Kostenabrechnung ist die Deutsche Gesetzliche Unfallversicherung (DGUV) als Verbindungsstelle für die Unfallversicherung betraut (§ 139 a SGB VII).

\section{Praktische Erfahrungen}

Die versicherungsrechtliche Einordnung einer Tätigkeit als Entsendung, Beschäftigungsverhältnis, oder selbständige Erwerbstätigkeit polnischer Staatsbürger beschäftigt die zuständigen Unfallversicherungsträger vor allem in Bezug auf das Bau-, Fleischerei-, Hotel- und Gaststättengewerbe, den Gesundheits- und Pflegesektor sowie die Landwirtschaft.

Ein Großteil der Saisonarbeitnehmer ist in der Landwirtschaft tätig. Aus den Statistiken der Agentur für Arbeit 2009 und 2010 ergibt sich, dass im Jahr 2009 insgesamt 184.241 Saisonarbeitnehmer ein Arbeitserlaubnisverfahren durchliefen, davon 179.710

17 Fuchs, in: ders. (Fußn. 11), Art. 36 Rdnr. 14. 
aus der Landwirtschaft. Im Jahr 2010 waren es 174.071 Saisonarbeitnehmer, davon 169.548 aus dem Bereich der Landwirtschaft. ${ }^{18}$

In Bezug auf Saisonarbeit, die von selbständig Erwerbstätigen aus Polen verrichtet wird, ist bei der Frage, ob eine Entsendung aus Polen vorliegt, von Bedeutung, ob es sich bei der vorübergehend in Deutschland ausgeübten Tätigkeit um eine ähnliche Tätigkeit wie bei der in Polen ausgeübten Tätigkeit handelt (Art. 12 Abs. 2 Verordnung (EG) Nr. 883/04). Unter einer ähnlichen Tätigkeit ist jede Tätigkeit zu verstehen, die im gleichen Sektor, etwa der Landwirtschaft, ausgeübt wird. Forstwirtschaft und Landwirtschaft hingegen sind grundsätzlich verschiedene Sektoren. ${ }^{19}$ Hilft also ein selbständiger Landwirt, der Viehzucht betreibt, vorübergehend in Deutschland bei der Obsternte, übt er eine ähnliche Tätigkeit aus, mit der Konsequenz, dass weiterhin die polnischen Sozialversicherungsvorschriften Anwendung finden. Hilft allerdings ein in Polen selbständiger Forstwirt bei der Obsternte in Deutschland, liegen verschiedene Tätigkeiten vor, so dass während der Saisonbeschäftigung die deutschen Vorschriften der Sozialversicherung und damit auch der gesetzlichen Unfallversicherung Anwendung finden. Die zuständigen landwirtschaftlichen Unfallversicherungsträger sind in der Praxis insbesondere mit Fallgestaltungen befasst, in denen es um Fragen der Anwendung polnischen oder deutschen Rechts geht sowie um Fragen im Zusammenhang mit der Sachleistungsaushilfe, die auch aufgrund unterschiedlicher Voraussetzungen in Bezug auf die Anerkennung eines Unfalls als Arbeitsunfall in beiden Ländern entstehen.

Des Weiteren sind die Unfallversicherungsträger häufig mit der Frage befasst, ob eine Tätigkeit als selbständige Erwerbstätigkeit oder abhängige Beschäftigung einzuordnen ist. ${ }^{20}$ Hiervon hängt, wenn keine Entsendung vorliegt und die deutschen Sozialversicherungsvorschriften Anwendung finden, ab, ob eine Versicherungspflicht in der gesetzlichen Unfallversicherung besteht, da Selbständige mit Ausnahme des Gesundheitsund Pflegesektors in der Regel nicht kraft Gesetz unfallversichert sind, für Beschäftigte hingegen Unfallversicherungsschutz kraft Gesetz besteht (§ 2 Abs. 1 Nr. 1 SGB VII).

Pflegekräfte aus Polen und anderen mittel- und osteuropäischen Ländern, sind, soweit die Regelungen der deutschen gesetzlichen Unfallversicherung auf sie Anwendung finden, entweder als Beschäftigte gemäß $§ 2$ Abs. 1 Nr. 1 SGB VII oder Selbständige gemäß $§ 2$ Abs. 1 Nr. 9 SGB VII kraft Gesetz versichert. Ob eine selbständige Erwerbstätigkeit ausgeübt wird, erfährt der zuständige Unfallversicherungsträger in der Regel über die Gewerbeanmeldung. Die Frage, ob im Einzelfall tatsächlich eine selbständige Tätigkeit oder eine Beschäftigung vorliegt ${ }^{21}$, stellt sich in Bezug auf Pflegekräfte in

$18 \mathrm{Vgl}$. Statistik der Bundesagentur für Arbeit Arbeitsmarkt in Zahlen, Arbeitsgenehmigungen-EU/Zustimmungen, Nürnberg, Berichtsjahre 2009 und 2010.

19 Information des Bundesministeriums für Ernährung, Landwirtschaft und Verbraucherschutz (BMELV) zur Sozialversicherungspflicht ausländischer Saisonarbeitskräfte, 2012.

20 Schannor (Fußn. 4), S. 442.

21 Hierzu umfassend aus sozialversicherungsrechtlicher Sicht: Körner, Pflegekräfte aus Osteuropa Licht ins Dunkel der Schwarzarbeit?, NZS 2011, S. 370 ff.; zur strafrechtlichen Bewertung, Urteil des AG München vom 10.11.2008, Az. 1115 OWi 298 Js 43552/07, Rdnr. 183 ff. 
Haushalten, insbesondere bei der 24 Stunden Pflege. Hiervon hängt die Zuständigkeit des Unfallversicherungsträgers $a^{22}$, aber auch, wer die Beiträge zur Unfallversicherung zu zahlen hat, die selbständige polnische Pflegekraft oder der die polnische Pflegekraft beschäftigende Haushalt. Die zuständigen Träger haben zu der gesamten Thematik Handbücher, Arbeitsanleitungen und Fragelisten entwickelt. In der Praxis entstehen häufig weitere Probleme, so etwa, wenn sich zwei polnische Pflegekräfte einen 24 Stunden Pflegetätigkeit teilen, der Haushalt (als Unternehmer) aber nur einen Beitrag zahlen möchte. Generell führt die Frage, wer letztlich die Beiträge zur gesetzlichen Unfallversicherung zu erbringen hat zu Problemen, hierzu zählt unter anderem die Realisierung der Beitragsansprüche. Verbesserungen und Vereinfachungen wären nach Einschätzung der betroffenen Träger durch Änderungen gesetzlicher Vorschriften zu erreichen.

\section{Charakteristika der gesetzlichen Unfallversicherung in Deutschland}

Finden aufgrund der Regelungen der Verordnung (EG) Nr. 883/04 die Vorschriften des deutschen Sozialversicherungsrechts Anwendung, sind polnische Arbeitnehmer über die Vorschriften der gesetzlichen Unfallversicherung gegen Arbeitsunfälle und Berufskrankheiten versichert.

Die gesetzliche Unfallversicherung ist ein eigenständiger Zweig des deutschen Sozialversicherungssystems. Träger der Unfallversicherung sind die Berufsgenossenschaften und Unfallversicherungsträger der öffentlichen Hand. Die Berufsgenossenschaften sind nach Branchen gegliedert, insoweit bestimmt sich die Zuständigkeit für ein Unternehmen und seine Beschäftigten nach der Branche, in der es tätig ist. Für Beschäftigte in Haushalten ist der kommunale Unfallversicherungsträger der öffentlichen Hand zustän$\operatorname{dig}(\S 129$ Abs. 1 Nr. 2 SGB VII).

Die Finanzierung der gesetzlichen Unfallversicherung erfolgt allein über Beiträge der Unternehmen ( $\$ 150$ SGB VII). Da das Gesetz die Unternehmen im Gegenzug ${ }^{23}$ von ihrer Haftung gegenüber den Arbeitnehmern befreit (§ 104 SGB VII), hat die gesetzliche Unfallversicherung einen umfassenden Leistungsauftrag, der Kompensationsfunktion hat.

Der gesetzliche Auftrag der Unfallversicherung besteht gemäß § 1 SGB VII darin,

„1. mit allen geeigneten Mitteln Arbeitsunfälle und Berufskrankheiten sowie arbeitsbedingte Gesundheitsgefahren zu verhüten,

2. nach Eintritt von Arbeitsunfällen oder Berufskrankheiten die Gesundheit und die Leistungsfähigkeit der Versicherten mit allen geeigneten Mitteln wiederherzustellen und sie oder ihre Hinterbliebenen durch Geldleistungen zu entschädigen.“

22 Lauterbach/Schwerdtfeger, in: Breuer (Fußn. 13), § 2 Rdnr. 343.

23 Lauterbach/Platz, in: Breuer (Fußn. 13), § 150, Rdnr. 4. 
Haben Versicherte einen Arbeitsunfall erlitten, so haben sie Anspruch auf Heilbehandlung einschließlich Leistungen zur medizinischen Rehabilitation, auf Leistungen zur Teilhabe am Arbeitsleben (berufliche Rehabilitation) und am Leben in der Gemeinschaft (soziale Rehabilitation), auf ergänzende Leistungen, auf Leistungen bei Pflegebedürftigkeit sowie auf Geldleistungen (§ 26 SGB VII). Die Durchführung der Heilbehandlung (§ 34 SGB VII) unterliegt bestimmten Verfahrens- und Qualitätsanforderungen. Ziel ist es angesichts der Kompensationsfunktion, den gesetzlichen Auftrag, die Gesundheit und Leistungsfähigkeit mit allen geeigneten Mitteln wiederherzustellen, zu erfüllen. Deswegen erfolgt die Behandlung nach einem Arbeitsunfall durch einen besonders qualifizierten und zugelassenen Arzt (Durchgangsarzt). Ebenso können nur Kliniken, die die besonderen Anforderungen, die an die Versorgung Unfallverletzter gestellt werden, an der medizinischen Versorgung teilnehmen. Zu den besonderen Verfahren der Heilbehandlung, die angewendet werden, zählen das Durchgangsarztverfahren, das Verletzungsartenverfahren zur stationären Behandlung schwerer Unfallverletzungen sowie auch die Berufsgenossenschaftliche stationäre Weiterbehandlung. ${ }^{24}$ Der gesamte Heilbehandlungsprozess wird durch den zuständigen Unfallversicherungsträger gesteuert. $^{25}$

Bei der Berufsgenossenschaftlichen Stationären Weiterbehandlung (BGSW) handelt es sich um ein stationäres Rehabilitationsverfahren im Anschluss an die Akutphase. Angewandt wird es unter anderem bei Verletzungen des Stütz- und Bewegungsapparates sowie peripheren Nervenverletzungen. Das Verfahren kombiniert verschiedene insbesondere physio- und ergotherapeutische Methoden im Rahmen einer intensiven stationären Nachbehandlung. ${ }^{26}$ Kliniken, die an diesem Verfahren teilnehmen möchten, müssen spezielle personelle, apparative und räumliche Anforderungen erfüllen. ${ }^{27}$

\section{E. Grenzübergreifende Versorgungsstrukturen}

Im Zuge des polnisch-deutschen Expertenaustausches zur medizinischen Versorgung Unfallverletzter entwickelte sich die Idee einer grenzübergreifenden Versorgung in der Phase der Nachbehandlung Unfallverletzter.

In Deutschland beschäftigten Polen, die entweder in Deutschland oder in Polen ihren Wohnsitz haben, soll es möglich sein, im Anschluss an die akute Behandlungsphase eine Rehabilitationsbehandlung in einer polnischen Rehabilitationseinrichtung in An-

24 Bereiter-Hahn/Mehrtens, Gesetzliche Unfallversicherung, 5. Auflage, § 34 Rdnr. 8 ff.

25 Ebd., § 34 Rdnr. 3.2 ff.

26 Ebd., § 34 Rdnr. 8.8.

27 Handlungsanleitung zur Verordnung, Durchführung und Qualitätssicherung der Physiotherapie/Krankengymnastik - Physikalischen Therapie, Erweiterten Ambulanten Physiotherapie (EAP), Berufsgenossenschaftlichen Stationären Weiterbehandlung (BGSW) und sonstigen stationären Maßnahmen, Januar 2008. S. 9-14. 
spruch zu nehmen, die nach den Verfahren und Qualitätsstandards erfolgt, wie sie das beschriebene Verfahren der Berufsgenossenschaftlichen Stationären Weiterbehandlung (BGSW) vorsieht.

Eine derartiges Versorgungskonzept bietet mehrere Vorteile: Der Heilungsprozess wird durch eine vertraute Umgebung gefördert; insbesondere die Sprache und kulturelle Gemeinsamkeiten fördern das Vertrauen in das medizinische Personal und können die Heilung positiv beeinflussen. Dem zuständigen deutschen Unfallversicherungsträger obliegt auch weiterhin die Steuerung des Verfahrens mit dem Ziel, einen optimalen Heilungserfolg zu erreichen. Die polnische Rehabilitationseinrichtung wiederum profitiert von der Leistungserbringung gegenüber dem deutschen Unfallversicherungsträger. Ebenso profitieren ggf. auch andere Patienten der Rehabilitationsklinik von einem erweiterten Leistungsangebot.

Ähnliche Möglichkeiten einer grenzübergreifenden medizinischen Versorgung, gibt es im Bereich der Krankenversicherung. So können deutsche Krankenversicherungsträger mit Einrichtungen im Ausland Verträge abschließen, die eine medizinische Versorgung nach den Grundsätzen der gesetzlichen Krankenversicherung vorsehen (§ $140 \mathrm{e}$ SGB V). Da der Versicherte die inländischen, im SGB V vorgesehenen Sachleistungen aufgrund eines entsprechenden Vertrags mit einer Einrichtung im Ausland erhält, ist diese Möglichkeit als „auf Vertrag gegründetes Sachleistungsprinzip“28 bezeichnet worden.

Bezogen auf die gesetzliche Unfallversicherung sieht $\S 34$ Abs. 8 Satz 1 SGB VII vor, dass die Beziehungen zwischen den Unfallversicherungsträgern und anderen als den in Absatz 3 genannten Stellen, die Heilbehandlung durchführen oder an ihrer Durchführung beteiligt sind, durch Verträge geregelt werden. Die Beziehungen der Träger der gesetzlichen Unfallversicherung sind damit nicht auf die in Absatz 3 erwähnten kassenärztlichen Bundesvereinigungen beschränkt. Generell ist es demnach den Unfallversicherungsträgern möglich, mit anderen Leistungsanbietern Verträge über die medizinische Versorgung Unfallverletzter zu schließen, soweit diese die besonderen Anforderungen der gesetzlichen Unfallversicherung erfüllen. In Bezug auf die Dienstleistungsfreiheit (Art. 56 AEUV) dürfte der Abschluss von Verträgen mit ausländischen Leistungsanbietern aus europarechtlicher Sicht keinen Bedenken begegnen.

\section{F. Regelungen im Europarecht}

Sowohl die Verordnung (EG) Nr. 883/04 als auch die Richtlinie 2011/24/EU enthalten Regelungen zur grenzübergreifenden medizinischen Versorgung.

28 Kingreen, Der Vorschlag der Europäischen Kommission für eine Patienten-Richtlinie, ZESAR 2009, S. 115. 


\section{Regelungen des koordinierenden Verordnungsrechts}

Nach den Regelungen der Verordnung (EG) Nr. 883/04 kann sich eine Person, die in einem Mitgliedstaat einen Arbeitsunfall erlitten hat, anschließend in einen anderen Mitgliedstaat begeben, um sich dort weiterbehandeln zu lassen (Art. 36 Absatz 1 i.V.m. Art. 17, 20 Verordnung (EG) Nr. 883/04). Hierfür bedarf sie, wenn sie sich nicht in ihren Wohnstaat begibt, der Genehmigung durch den zuständigen Träger (Art. 20 Verordnung (EG) Nr. 883/04). In Polen erhält sie über das bereits beschriebene Verfahren der Sachleistungsaushilfe Leistungen über den zuständigen polnischen Sozialversicherungsträger nach den Vorschriften der polnischen Sozialversicherung.

Die Behandlung eines Unfallverletzten in Polen nach den Vorschriften der gesetzlichen Unfallversicherung in Deutschland sieht die Verordnung nicht vor.

\section{Patientenrichtlinie}

Der Europäische Gerichtshof (EuGH) hat mit seiner durch die Urteile Kohll und Decker $^{29}$ eingeleiteten Rechtsprechung besondere Rechte von Patienten in Bezug auf die Inanspruchnahme grenzübergreifender Behandlungen etabliert. Danach erlaubt es die passive Dienstleistungsfreiheit als Ausfluss der Dienstleistungsfreiheit gemäß Art. 56 AEUV einem Patienten, sich zu einer medizinischen Behandlung in einen anderen Mitgliedstaat zu begeben, und sich im Anschluss die Kosten durch den zuständigen Träger erstatten zu lassen. Zwischenzeitig hat die ständig weiter entwickelte Rechtsprechung in der Richtlinie 2011/24/EU vom 9. März 2011 über die Ausübung der Patientenrechte in der grenzüberschreitenden Gesundheitsversorgung einen rechtlichen Rahmen gefunden. Die Behandlung im Ausland erfolgt danach nach den Vorschriften des Behandlungsstaates (Art 4 Richtlinie 2011/24/EU). Zwar dürfen nationale Steuerungsverfahren auch bei der Behandlung im Ausland vorgeschrieben werden (Erwägungsgrund 37 Richtlinie 2011/24/EU), allerdings sieht die Richtlinie nur die Möglichkeit der Kostenerstattung vor. Das grenzübergreifende Versorgungskonzept in der gesetzlichen Unfallversicherung ist hingegen auf die Erbringung einer Sachleistung ausgerichtet.

Jedoch lassen sowohl die Rechtsprechung des EuGH sowie die Richtlinie 2011/24/EU den Ansatz erkennen, grenzübergreifende medizinische Versorgungsstrukturen nicht auf die in der Verordnung (Nr.) 883/04 vorgesehenen Verfahren begrenzen zu wollen.

Das grenzübergreifende Versorgungskonzept der Unfallversicherung stünde dem Versicherten damit als weitere Möglichkeit zur Verfügung, grenzübergreifend medizinische Leistungen in Anspruch zu nehmen. Um einen eigenen Anspruch dürfte es sich

29 EuGH-Urteil v. 28.4.1998, Rs. C-158/96 (Kohll), Slg. 1998, I-1931; v. 28.4.1998, Rs. C-120/95 (Decker), Slg. 1998, I-1831. 
dabei nicht handeln, da letztlich dem Versicherten durch einen ausländischen Erbringer Leistungen nach den Vorschriften der gesetzlichen Unfallversicherung (SGB VII) gewährt werden sollen. ${ }^{30}$

\section{G. Anforderungen an die Umsetzung}

Bei der Umsetzung eines grenzübergreifenden Versorgungskonzepts, das eine Rehabilitationsbehandlung im Anschluss an die akute Behandlung in einer polnischen Rehabilitationsklinik nach den Maßstäben der gesetzlichen Unfallversicherung in Deutschland vorsieht, sind folgende Punkte zu beachten:

Der Leistungsrahmen und die Qualitäts- sowie Verfahrensanforderungen sollten den Bestimmungen des $\S 34$ SGB VII entsprechen und klar definiert sein. Das ärztliche und medizinische Personal in Polen sollte in den anzuwendenden Verfahren geschult sein. Des Weiteren ist zu gewährleisten, dass der zuständige Unfallversicherungsträger das Heilverfahren weiterhin steuert. Dies erfordert Austausch und Kontakt mit dem zuständigen ärztlichen und medizinischen Personal in Polen.

Organisatorische Details sowie Verfahrens-, Vergütungs- und Haftungsfragen sollten darüber hinaus mit dem Leistungsanbieter im Vorfeld geklärt sein.

Auch europarechtliche Vorgaben, insbesondere Aspekte der Dienstleistungsfreiheit (Art 56 AEUV), sind zu beachten. Danach sollte es sich um ein freiwilliges Angebot an Versicherte handeln, um ihnen zustehende Rechte aufgrund der Verordnung (EG) Nr. 883/04 sowie der Patientenrichtlinie (2011/24/EU) nicht einzuschränken. Eine eingeschränkte Wahlfreiheit bezüglich der Rehabilitationsklinik in Polen dürfte, soweit sie aufgrund der besonderen Qualitätsanforderungen in der Unfallversicherung erfolgt, hingegen gerechtfertigt sein. Eine Begrenzung auf Kooperationen mit Leistungsanbietern, die die besonderen Verfahrens- und Qualitätsanforderungen der gesetzlichen Unfallversicherung erfüllen, dürfte ebenfalls gerechtfertigt sein. Gemäß dem Grundsatz der Nichtdiskriminierung (Art 18 AEUV) sollte das Angebot nicht nur polnischen Staatsbürgern zur Verfügung stehen.

\section{H. Fazit}

Die Arbeitnehmerfreizügigkeit im deutsch-polnischen Kontext wirkt sich auch auf die gesetzliche Unfallversicherung in Deutschland aus.

$30 \mathrm{Zu}$ den Ansprüchen gem. $\S 140$ e SGB V: Kingreen, Ein neuer rechtlicher Rahmen für einen Binnenmarkt für Gesundheitsdienstleistungen, NZS 2005, S. 507. 
Die deutschen Unfallversicherungsträger sind in der Praxis insbesondere mit der versicherungsrechtlichen Bewertung von Beschäftigungsverhältnissen und selbständigen Tätigkeiten in Bezug auf Arbeitskräfte aus Polen befasst.

Finden die Vorschriften der gesetzlichen Unfallversicherung Anwendung und erleidet ein polnischer Versicherter in Deutschland einen Arbeitsunfall, so könnte ein grenznahes Versorgungsangebot es ermöglichen, Rehabilitationsleistungen der gesetzlichen Unfallversicherung durch eine polnische Einrichtung in Anspruch zu nehmen.

Die grenzübergreifende Versorgung bietet folgende Vorteile: Versicherte haben die Möglichkeit einer heimatnahen Versorgung, der zuständige Unfallversicherungsträger steuert weiterhin das Heilverfahren und die Rehabilitationseinrichtung erweitert ggf. ihr Leistungsspektrum.

Ein derartiges Angebot kann die Mobilität von Arbeitskräften und somit die Arbeitnehmerfreizügigkeit fördern. 


\title{
Familienleistungen bei grenzüberschreitenden Sachverhalten
}

\author{
Renata Babińska-Górecka und Karolina Stopka
}

A. Einführung 285

B. Begriff und Koordinierung der Familienleistungen 287

I. Begriff der Familienleistungen nach polnischem Recht 287

II. Anwendbarkeit bei grenzüberschreitenden Sachverhalten 289

III. Zur Frage der Kumulierung 291

IV. Begriff der Familienleistungen nach Unionsrecht 293

V. Folgerungen für die Qualifizierung nach polnischem Recht 294

C. Weitere Problemstellungen 297

\section{A. Einführung}

Familienleistungen wurden in den Gesetzgebungen aller EU-Mitgliedsstaaten vorgesehen und gehören zu den häufigsten Leistungen, die von den Angestellten innerhalb der Union und dem Europäischen Wirtschaftsraum in Anspruch genommen werden. ${ }^{1}$ Außerdem beeinflusst die Erwerbsmigration eines Familienmitgliedes auf der Ebene der Familienleistungen - im Gegensatz zu anderen, von der Koordinierung der Sozialversicherungssysteme umfassten Leistungen - unmittelbar den Rechteumfang anderer Familienmitglieder. Demzufolge gewinnt die Regelung von Koordinierungsgrundsätzen derartiger Leistungen eine besondere Bedeutung. ${ }^{2}$ Die Entscheidung über Koordinierungsangelegenheiten in Bezug auf die Familienleistungen erfolgt durch Anwendung einer Reihe rechtlicher Regelungen, die in der Verordnung Nr. 883/2004³ sowie in nationalen

1 G. Uścińska, Koordynacja świadczeń rodzinnych w regulacjach wspólnotowych, in: Polityka Społeczna Nr. 8 2007, S. 45; M. Rusewicz, Zasady nabywania świadczeń rodzinnych w Unii Europejskiej, in: Prawo i Podatki Unii Europejskiej Nr. 5 2007, S. 7.

2 G. Uścińska, Konsekwencje integracji europejskiej dla ubezpieczeń społecznych, in: Ewolucja ubezpieczeń społecznych w okresie transformacji ustrojowej, Bydgoszcz 2011, S. 41-42; L. Mitrus, Zakres przedmiotowy koordynacji systemów zabezpieczenia społecznego w Unii Europejskiej, in: Z zagadnień prawa pracy i prawa socjalnego. Księga jubileuszowa Profesora Herberta Szurgacza, Warszawa 2011, S. 500; E. Eichenhofer, Sozialrecht der Europäischen Union, Berlin 2006, S. 188.

3 Verordnung (EG) des Europäischen Parlaments und des Rates vom 29. April 2004 zur Koordinierung der Systeme der sozialen Sicherheit, Dz.U. UE. L.04.166.1.; nachstehend: Verordnung Nr. $883 / 2004$. 